\title{
ON A COMMON GENERALIZATION OF BORSUK'S AND RADON'S THEOREM
}

By

E. G. BAJMÓCZY and I. BÁRÁNY (Budapest)

1. The well-known theorem of RADON [3] says that if $A \subset R^{d}$ and $|A| \geqq d+2$, then there exist $B, C \subset A, B \cap C=\varnothing$ such that conv $B \cap$ conv $C$ is not empty. It is clear that for each finite set $A=\left\{a_{1}, \ldots, a_{n}\right\}$ in $R^{d}$ with $n \geqslant d+2$ one can find a linear map $f: R^{d+1} \rightarrow R^{d}$ and a set $A^{\prime}=\left\{a_{1}^{\prime}, \ldots, a_{n}^{\prime}\right\} \subset R^{d+1}$ such that $f\left(a_{i}^{\prime}\right)=a_{i}$ $i=1,2, \ldots, n$ and int conv $A^{\prime}$ is not empty and vert conv $A^{\prime}=A^{\prime}$. In view of this fact, Radon's theorem can be stated in the following way.

RADON's THEOREM. Let $P \subset R^{d+1}$ be a convex polytope with non-empty interior. Put $A=$ vert $P$. If $f: R^{d+1} \rightarrow R^{d}$ is a linear map, then there exist two disjoint sets $B, C \subset A$ such that $f(\operatorname{conv} B) \cap f(\operatorname{conv} C)$ is non-empty.

The surprising fact here is that the word "linear" can be replaced by "continuous", namely, a continuous analogue of Radon's theorem is true;

THEOREM 1. Let $P \subset R^{d+1}$ be a convex polytope with non-empty interior. Given an $f: \partial P \rightarrow R^{d}$ continuous map, there exist two disjoint faces, $B$ and $C$, of $P$ such that $f(B) \cap f(C) \neq \varnothing$.

COROllary. Let $T$ be $a(d+1)$-dimensional simplex. Denote its d-faces by $L_{1}, L_{2}, \ldots, L_{d+2}$. If $f: \partial T \rightarrow R^{d}$ is a continuous map, then $\bigcap_{i=1}^{d+2} f\left(L_{i}\right)$ is non-empty.

If $f$ is a linear map, then this statement is an easy consequence (in fact, equivalent) of Helly's theorem (see [3]). The interesting fact here is that in this particular case a continuous version of Helly's theorem holds true.

Let us now introduce some notions. Given a convex compact set $C \subset R^{d+1}$ with non-empty interior and a vector $a \in R^{d+1}, a \neq 0$, we write

$$
C(a)=\left\{x \in C:\langle a, x\rangle=\max _{t \in C}\langle a, t\rangle\right\} \text {. }
$$

Two points, $x$ and $y$, of $C$ are said to be opposite if for some $a \in R^{d+1}, x \in C(a)$ and $y \in C(-a)$. If $C$ happens to be a polytope, then $C(a)$ is a proper face of $C$. In this case we say that the two faces $C(a)$ and $C(-a)$ are opposite.

THEOREM 2. Given a polytope $P \subset R^{d+1}$ with non-empty interior and a continuous map $f: \partial P \rightarrow R^{d}$, there exist two opposite faces, $B$ and $C$, of $P$ such that $f(B) \cap f(C)$ is non-empty.

It is evident that opposite faces of $P$ are disjoint. Thus Theorem 2 implies Theorem 1.

Speaking about points instead of faces Theorem 2 can be formulated as follows. 
THEOREM 2'. Given a polytope $P \subset R^{d+1}$ with non-empty interior and a continuous map $f: \partial P \rightarrow R^{d}$, there exist two opposite points, $x$ and $y$, of $P$ with $f(x)=f(y)$.

We shall prove this Theorem $2^{\prime}$ which yields a generalization of Borsuk's theorem [1]. In order to state Borsuk's theorem put $S^{d}=\left\{x \in R^{d+1}:\|x\|=1\right\}$.

BORSUK'S THEOREM. If $f: S^{d} \rightarrow R^{d}$ is a continuous map, then there is a point $x \in S^{d}$ with $f(x)=f(-x)$.

THEOREM 3. Let $C \subset R^{d+1}$ be a convex compact set with nonempty interior. If $f: \partial C \rightarrow R^{d}$ is a continuous map, then there exist two opposite points, $x$ and $y$, of $C$ with $f(x)=f(y)$.

Again, Theorem 3 implies Theorem 2'. However, we shall get Theorem 3 from Theorem 2 ' by a simple continuity argument.

Further, our Theorem 3 contains Borsuk's theorem (put simply $C=$ conv $S^{d}$ ). On the other hand, Theorem $2^{\prime}$ is proved using Borsuk's theorem.

2. We need a simple proposition.

Proposition. If $P$ is a polytope in $R^{d}$ and $x, y, x_{n} \in P \quad n=1,2, \ldots$ and $\lim x_{n}=x$, then there is an $\varepsilon>0$ and $N$ such that $x_{n}+\varepsilon \cdot(y-x) \in P$ for $n>N$.

Proof. This proposition is true for any cone $C$ (instead of $P$ ) whose vertex is $x$ (with arbitrary $\varepsilon>0$ and $n$ ), so it is true for $C \cap B(x, \delta)$ where $B(x, \delta)$ is the ball with center $x$ and radius $\delta$. But $P \cap B(x, \delta)=C \cap B(x, \delta)$ for a sufficiently small $\delta>0$ where

$$
C=\left\{z \in R^{d}: z=x+\lambda(w-x), \lambda>0, w \in P\right\}
$$

is a cone with vertex $x$.

Proof of Theorem 2'. Put $Q=P-P$. $Q$ is a polytope with non-empty interior. It is centrally symmetric with respect to the origin. For $x \in Q$ write

$$
h(x)=\max \{z: x=z-w, z, w \in P\}
$$

where max is meant in the lexicographic ordering of $R^{d+1}$. Clearly $h: Q \rightarrow P$ is welldefined. An easy computation shows that the vector $w$ corresponding to $z=h(x)$ equals $h(-x)$.

We claim that $h$ is continuous. Indeed, let $x, x_{n} \in Q, x=\lim x_{n}$ and $x_{n}=z_{n}-w_{n}$ where $z_{n}=h\left(x_{n}\right)$. We can choose a subsequence $n_{i}$ so that $z_{n_{i}}$ and, consequently $w_{n_{i}}$ converge. Put $z=\lim z_{n_{i}}$ and $w=\lim w_{n_{i}}$; clearly $x=z-w$. We claim that $z=h(x)$. If not, then $z<h(x)$ in the lexicographic ordering. By the Proposition, for a sufficiently small positive $\varepsilon$ and large $i$

$$
z^{\prime}=z_{n_{i}}+\varepsilon(h(x)-z) \in P \quad \text { and } \quad w^{\prime}=w_{n_{i}}+\varepsilon(h(-x)-w) \in P .
$$

Now $z^{\prime}-w^{\prime}=x_{n_{i}}$ and $z^{\prime}>z_{n_{i}}$ contradicting $z_{n_{i}}=h\left(x_{n_{i}}\right)$. This means that $z=h(x)$. Thus, every convergent subsequence of $z_{n}$ tends to $h(x)$. Now by compactness $\lim z_{n}=h(x)$, i.e., $h$ is continuous. 
Next we claim that $x \in Q(a)$ implies $h(x) \in P(a)$ and $h(-x) \in P(-a)$. Indeed, if $x \in Q(a)$ then $\max _{t \in Q}\langle a, t\rangle=\langle a, x\rangle$. Of course, $x=h(x)-h(-x)$ and $h(x), h(-x) \in P$. Whence

$$
\begin{gathered}
\langle a, h(x)\rangle+\langle-a, h(-x)\rangle=\langle a, x\rangle=\max _{t \in Q}\langle a, t\rangle= \\
=\max _{u, v \in P}\langle a, u-v\rangle=\max _{u \in P}\langle a, u\rangle+\max _{v \in P}\langle-a, v\rangle
\end{gathered}
$$

and so $h(x) \in P(a)$ and $h(-x) \in P(-a)$. This further implies that for $x \in \partial Q h(x)$ and $h(-x)$ belong to $\partial P$.

Now we define a map $g: \partial Q \rightarrow R^{d}$ in the following way: for $x \in \partial Q$ let $g(x)=$ $=f(h(x))$. This map is welldefined and continuous. Let us observe now that the conditions of Borsuk's theorem are fulfilled for the map $g$ (instead of $S^{d}$ we have $\partial Q$ here but this is indifferent). In this case Borsuk's theorem says that there is a point $x \in \partial Q$ with $g(x)=g(-x)$. Now there exists $a \in R^{d+1}, a \neq 0$ such that $x \in Q(a)$. Then $z=h(x) \in P(a)$ and $w=h(-x) \in P(-a)$, i.e., $z$ and $w$ are opposite points of $P$ and $f(z)=f(h(x))=g(x)=g(-x)=f(h(-x))=f(w)$. And this is what we wanted to prove.

Proof of the Corollary. It is easy to check that if $B$ and $C$ are disjoint faces of the simplex $T$, then for any $i=1,2, \ldots, d+2$ either $B \subset L_{i}$ or $C \subset L_{i}$ (or both). This fact proves the Corollary.

PROOF OF THEOREM 3. Without loss of generality we may suppose that $0 \in \operatorname{in} t C$.

Now let $P$ be a polytope inscribed in $C$, i.e., vert $P \subset \partial C$ and suppose further that 0 int $P$. Then a continuous map $f_{P}: \partial P \rightarrow R^{d}$ can be defined as $f_{P}(x)=f(\hat{\gamma} x)$, where $\lambda$ is the unique positive number with $\lambda x \in \partial C$. By Theorem $2^{\prime}$, there are opposite points of $P, z_{P}$ and $w_{P}$ with $f_{P}\left(z_{P}\right)=f_{P}\left(w_{P}\right)$.

Now choose a sequence of inscribed polytopes $P_{1}, P_{2}, \ldots$ with $0 \in$ int $P_{n}$. Suppose further that vert $P_{n} \subset$ vert $P_{n+1}$ and $\partial C \cap \bigcup_{1}^{\infty} P_{n}$ is dense in $\partial C$. Again, for each $n$ there exist opposite (for $P_{n}$ ) points $z_{n}$ and $w_{n}$ with $f_{n}\left(z_{n}\right)=f_{n}\left(w_{n}\right)$. where $f_{n}=f_{P_{n}}$. Since $z_{n}$ and $w_{n}$ are opposite poiats in $P_{n}$ there is a vector $a_{n} \in S^{d}$ such that $z_{n} \in P_{n}\left(a_{n}\right)$ and $w_{n} \in P_{n}\left(-a_{n}\right)$.

By the compactness of $C$ and $S^{d}$ we may suppose that $z_{i n}, w_{n}$ and $a_{n}$ converge, their limits are $z, w \in \partial C$ and $a \in S^{d}$ respectively. It is easy to see that $z$ and $w$ are opposite points of $C$ (with normal $a$ ) and $f(z)=f(w)$.

3. REMARKS. 1. Theorem 1 can be interpreted in the following way. Let $P \subset R^{d+1}$ be a convex polytope with non-empty interior. Then it is not possible to make a drawing of $\partial P$ in $R^{d}$ so that disjoint faces of $P$ be disjoint in the drawing.

2. We can give a second proof of Theorem 2 which is more involved than the above one but does not make use of Borsuk's theorem. It relies on a suitably modified version of the main lemma of [2].

3. The following generalization of Theorem 3 holds true.

THEOREM 4. Let $C \subset R^{d+1}$ be a convex compact set with non-empty interior. Let $f$ be a point to set map from $\partial C$ to the family of all compact convex subsets of a compact set of $R^{d}$. If $f$ is upper semi-contimuous (i.e., $x_{n} \rightarrow x, y_{n} \in f\left(x_{n}\right)$, and $y_{n} \rightarrow y$ 
implies $y \in f(x))$, then there exist two opposite points, $z$ and $w$, of $C$ with $f(z) \cap f(w) \neq \varnothing$.

This theorem follows from Theorem 3 nearly the same way as Kakutani's fixed-point theorem follows from Brouwer's one.

4. We conclude with a conjecture. There is a generalization of Radon's theorem which is due to H. TVERBERG [5]. In the spirit of our formulation of Radon's theorem this generalization runs as follows:

THEOREM. Let $P \subset R^{n}$ be a convex polytope with non-empty interior. Here $n=(r-1)(d+1)$, Given an $f: R^{n} \rightarrow R^{d}$ linear map there are disjoint proper faces $B_{1}, B_{2}, \ldots, B_{r}$ of $P$, such that $\bigcap_{i=1}^{r} f\left(B_{i}\right)$ is non-empty.

We think (but can neither prove nor disprove) that in this theorem it is enough to assume that $f: \partial P \rightarrow R^{d}$ is a continuous map.

Acknowledgement. We are indebted to Prof. M. Bognár for his valuable comments on an earlier version of this paper and to $L$. Lovász for the question that led us from Theorem $2^{\prime}$ to Theorem 3.

\section{References}

[1] P. Alexandroff, H. Hopf, Topologie, Springer (Berlin, 1935).

[2] I. BÁRÁNY, An algorithm to compute fixed points (unpublished).

[3] B. GrünBaum, Convex Polytopes, Interscience (London, 1967).

[4] S. KakUTANI, A generalization of Brouwer's fixed-point theorem, Duke Math. J., 8 (1941), 457 - 458 .

[5] H. TVerberg, A generalization of Radon's theorem, J. London Math. Soc., 41 (1966), 123128.

(Received February 17, 1978)

OTSZK, COORDINATION AND SCIENTIFIC SECRETARIAT

1132 BUDAPEST, VICTOR HUGO U. $18-22$. 